\title{
Interval Observers for Time-Varying Discrete-Time Systems
}

\author{
Denis Efimov, Wilfrid Perruquetti, Tarek Raïssi, Ali Zolghadri
}

\begin{abstract}
This paper deals with interval state observer design for timevarying discrete-time systems. The problem of a similarity transformation computation which connects a (time-varying) matrix and its nonnegative representation is studied. Three solutions are proposed: for a generic time-varying system, a system with positive state, and for a particular class of periodical systems. Numerical simulations are provided to demonstrate advantages of the developed techniques.
\end{abstract}

\section{INTRODUCTION}

Observer design is a central problem in many engineering applications where estimated states or outputs are required for designing control laws or monitoring system variables. Many important contributions are available in the literature, see for instance and among others [1], [2], [3], [4] and the references therein. When it deals with uncertain and disturbed systems, the classical nonlinear local filtering techniques (for example, the Extended Kalman Filter and its variants) could be useful to solve the estimation problem (see for instance [5]). However it is well known that, in general, only local convergence can be expected [6]. If the uncertainty cannot be neglected, another alternative solution based on the set-membership (or interval) estimation has been recently developed to solve the observation problem for systems subject to bounded perturbations. The basic idea is to compute the set of admissible values for the state at each instant of time.

There exist many interval observers proposed for continuous-time (linear and nonlinear) systems based on the monotone systems theory [7], [8], [9], [10], [11], [12]. One of the most restrictive assumptions for design of interval observers corresponds to cooperativity of the estimation error dynamics. To overcome this drawback, it has been recently shown that under some mild conditions, by applying a similarity transformation, a Hurwitz matrix can be transformed into a Hurwitz and Metzler (cooperative) matrix [9], [13], [10]. This transformation is time varying in [9], [13], while in [10] the transformation matrix is constant and real (a constructive procedure for calculation of the transformation matrix is also given in [10]). In [14] this result has been extended to the class of time-varying systems, where constant and time-varying similarity transformations have been proposed to represent a time-varying interval matrix in a Metzler form.

For discrete-time models, several set-membership state estimators have been also developed using simple geometrical forms (parallelotopes, ellipsoids, zonotopes or intervals) [15], [16], [17], [18], [19], [20]. The solutions are based on the well-known prediction/correction approach (also called open-loop observers, framers or predictors, where the system equations are solved starting from a set of initial conditions taking on each step the values consistent with the output measurements). The main drawback of these techniques is that the convergence rate cannot be tuned since no observer gain is used. In this paper, we propose to extend the interval observer methodology,

The first two authors are with the Non-A project at INRIA - LNE, Parc Scientifique de la Haute Borne, 40 avenue Halley, Bât.A Park Plaza, 59650 Villeneuve d'Ascq, France, \{denis.efimov; wilfrid.perruquetti\}@inria.fr.

The third author is with Conservatoire National des Arts et Métiers (CNAM), Rue St-Martin, 75141 Paris, tarek.raissi@cnam.fr.

The last author is with University of Bordeaux, IMS-lab, Automatic control group, 351 cours de la libération, 33405 Talence, France, Ali.Zolghadri@imsbordeaux.fr. initially developed for continuous-time systems [7], [13], [10], to uncertain discrete-time systems. The recent papers [21], [22] have been devoted to constructions of closed-loop interval observers for Linear Time-Invariant (LTI) discrete-time systems. In this work we will focus our attention on the time-varying case, which is much more complex from both analysis and design points of view.

The main contribution of this work is to extend the approach of interval observer design [21], [10] to Linear Time-Varying (LTV) discrete-time systems described by

$$
\begin{gathered}
x(t+1)=A(t) x(t)+b(t), \\
y(t)=C(t) x(t)+v(t), t \in \mathbb{Z}_{+},
\end{gathered}
$$

where $x(t) \in \mathbb{R}^{n}$ and $y(t) \in \mathbb{R}^{p}$ are the state and the output signal available for measurements; $b: \mathbb{Z}_{+} \rightarrow \mathbb{R}^{n}$ is an unknown input; $v$ : $\mathbb{Z}_{+} \rightarrow \mathbb{R}^{p}$ is the measurement noise, the matrix functions $A: \mathbb{Z}_{+} \rightarrow$ $\mathbb{R}^{n \times n}, C: \mathbb{Z}_{+} \rightarrow \mathbb{R}^{p \times n}$ of appropriate dimensions are given (the real and integer numbers are denoted by $\mathbb{R}$ and $\mathbb{Z}$ respectively, $\mathbb{R}_{+}=$ $\{\tau \in \mathbb{R}: \tau \geq 0\}$ and $\mathbb{Z}_{+}=\mathbb{Z} \cap \mathbb{R}_{+}$). In order to ensure cooperativity of the estimation error, a similarity transformation of a stable (timevarying) matrix to a stable and nonnegative (time-varying) matrix has to be constructed. Both constant and time-varying periodic similarity transformations are investigated.

The paper is organized as follows. Some basic facts from the theory of interval estimation are given in Section 2. The main result is described in Section 3. Two examples of computer simulation are presented in Section 4: a third order time-varying periodical system and the repressilator (a model from biology).

\section{PRELIMINARIES}

Euclidean norm of a vector $x \in \mathbb{R}^{n}$ will be denoted by $|x|$, and for a measurable and locally essentially bounded input $u: \mathbb{Z} \rightarrow \mathbb{R}$ the symbol $\|u\|_{\left[t_{0}, t_{1}\right]}$ denotes its $L_{\infty}$ norm $\|u\|_{\left[t_{0}, t_{1}\right]}=\sup \{|u(t)|, t \in$ $\left.\left[t_{0}, t_{1}\right]\right\}$, if $t_{1}=+\infty$, then we will simply write $\|u\|$. We will denote by $\mathcal{L}_{\infty}$ the set of all inputs $u$ with the property $\|u\|<\infty$. Denote the sequence of integers $1, \ldots, k$ by $\overline{1, k}$. The symbols $I_{n}$ and $E_{n \times m}$ denote the identity matrix and the matrix with all entries equal 1 respectively (with dimensions $n \times n$ and $n \times m$ ). For a matrix $A \in \mathbb{R}^{n \times n}$, we denote respectively by $\lambda(A)$ the vector of its eigenvalues, $\|A\|_{\max }=\max _{i=\overline{1, n}, j=\overline{1, n}}\left|A_{i, j}\right|$ is the elementwise maximum norm (which is not sub-multiplicative) and $\|A\|_{2}=\sqrt{\max _{i=\overline{1, n}} \lambda_{i}\left(A^{\mathrm{T}} A\right)}$ is the induced $L_{2}$ matrix norm. For these norms the following relations are satisfied:

$$
\|A\|_{\max } \leq\|A\|_{2} \leq n\|A\|_{\max }
$$

The relation $P \succ 0(P \succeq 0)$ means that the matrix $P \in \mathbb{R}^{n \times n}$ is positive (nonnegative) definite.

\section{A. Interval analysis}

For two vectors $x_{1}, x_{2} \in \mathbb{R}^{n}$ or matrices $A_{1}, A_{2} \in \mathbb{R}^{n \times n}$, the relations $x_{1} \leq x_{2}$ and $A_{1} \leq A_{2}$ are understood elementwise. Given a matrix $A \in \mathbb{R}^{m \times n}$, define $A_{i, j}^{+}=\max \left\{0, A_{i, j}\right\}$ for all $i=\overline{1, n}$ and $j=\overline{1, n}$, and $A^{-}=A^{+}-A$. Let $x, \underline{x}, \bar{x} \in \mathbb{R}^{n}$ be vectors satisfying $\underline{x} \leq x \leq \bar{x}$ and $A \in \mathbb{R}^{m \times n}$ be a constant matrix, then

$$
A^{+} \underline{x}-A^{-} \bar{x} \leq A x \leq A^{+} \bar{x}-A^{-} \underline{x} .
$$

Since $A x=\left(A^{+}-A^{-}\right) x$, then for $\underline{x} \leq x \leq \bar{x}$ we obtain (3). 


\section{B. Cooperative discrete-time linear systems}

A matrix $A \in \mathbb{R}^{n \times n}$ is called Schur stable if all its eigenvalues have the norm less than one, it is called nonnegative if all its elements are nonnegative, and it is called Metzler if all its offdiagonal elements are nonnegative. Any solution of the system

$$
x(t+1)=A x(t)+\omega(t), \omega: \mathbb{Z}_{+} \rightarrow \mathbb{R}_{+}^{n}, t \in \mathbb{Z}_{+},
$$

with $x \in \mathbb{R}^{n}$ and a nonnegative matrix $A \in \mathbb{R}_{+}^{n \times n}$, is elementwise nonnegative for all $t \geq 0$ provided that $x(0) \geq 0$ [23]. Such systems are called cooperative (monotone) [23].

In the sequel we are interested in a Luenberger-like observer design with the gain $L$ such that the matrix $A-L C$ (the closed loop matrix of the estimation error dynamics) would be Schur stable and nonnegative. Usually it is not possible to find such a matrix $L$. However a change of variables $z(t)=S x(t)$ with a nonsingular matrix $S$ can be proposed such that, in the new coordinates, the matrix $S(A-L C) S^{-1}$ would satisfy the required properties. An idea how to design such a matrix $S$ is given in the lemma below.

Lemma 1. [10] Given the matrices $A \in \mathbb{R}^{n \times n}, R \in \mathbb{R}^{n \times n}$ and $C \in \mathbb{R}^{p \times n}$. If there exists a matrix $L \in \mathbb{R}^{n \times p}$ such that $\lambda(A-L C)=$ $\lambda(R)$, and there exist vectors $\varrho_{1} \in \mathbb{R}^{1 \times n}, \varrho_{2} \in \mathbb{R}^{1 \times n}$ such that the pairs $\left(A-L C, \varrho_{1}\right)$ and $\left(R, \varrho_{2}\right)$ are observable, then there is a nonsingular $S \in \mathbb{R}^{n \times n}$ such that $R=S(A-L C) S^{-1}$.

This result was used in [10] to design interval observers for continuous-time LTI systems with a Metzler matrix $R$. The main difficulty is to prove existence of a real and nonsingular matrix $S$, and to provide a constructive approach of its calculation. In [10] the matrix $S=O_{R} O_{A-L C}^{-1}$, where $O_{A-L C}$ and $O_{R}$ are respectively the observability matrices of the pairs $\left(A-L C, \varrho_{1}\right)$ and $\left(R, \varrho_{2}\right)$. Another (more strict) condition is that for $p=1$ the pair $(A, C)$ is observable (in this case there exists a matrix $L$ such that $\lambda(A) \neq \lambda(A-L C)=\lambda(R)$, that is equivalent to the existence of a unique $S=O_{R} O_{A-L C}^{-1}$ [24], where $O_{A-L C}$ and $O_{R}$ are observability matrices of $(A-L C, C)$ and $\left(R, \varrho_{2}\right)$ respectively). In addition, for any fixed matrices $A, R, C$ the equation $S A-R S=Q C$ is linear with respect to unknowns $S$ and $Q$, and it can be solved using an LMI routine. In the present work we will apply Lemma 1 to a nonnegative matrix $R$. Note that if the matrix $A-L C$ has only real positive eigenvalues, then $R$ can be chosen as diagonal or Jordan representation of $A-L C$.

The application of Lemma 1 is related to the inverse eigenvalue problem for nonnegative matrices (i.e. the problem of existence of a nonnegative matrix $R$ with the predefined eigenvalues $\lambda(A-L C)$ ), see the monograph [25] (section 11.2) for the necessary and sufficient conditions, which have to be imposed on $\lambda(A-L C)$ in order that a nonnegative $R$ exists. In [26] the fast Fourier transformation is used to design a real symmetric $R$ with a given vector of eigenvalues.

\section{Floquet theory for discrete-time systems}

Consider the LTV discrete-time system

$$
x(t+1)=A(t) x(t),
$$

where $x(t) \in \mathbb{R}^{n}$ is the state and $A: \mathbb{Z}_{+} \rightarrow \mathbb{R}^{n \times n}$. The system (4) is called reducible if there exists a matrix sequence $G: \mathbb{Z}_{+} \rightarrow \mathbb{R}^{n \times n}$ such that for all $t \in \mathbb{Z}_{+}$

$$
\begin{gathered}
G^{-1}(t+1) A(t) G(t)=N, \operatorname{det}\{G(t)\} \neq 0, \\
\sup _{t \in \mathbb{Z}_{+}}\|G(t)\|_{2}+\left\|G^{-1}(t)\right\|_{2}<+\infty
\end{gathered}
$$

for some matrix $N \in \mathbb{R}^{n \times n}$. In other words, if the system (4) is reducible, then the time-varying transformation of coordinates $z(t)=$
$G^{-1}(t) x(t)$ represents the system in the time-invariant form

$$
z(t+1)=N z(t)
$$

Since $G(t)$ and $G^{-1}(t)$ are bounded, stability of (4) follows (5).

The system (4) is called periodical if there exists a $\tau>1$ such that $A(t+\tau)=A(t)$ for all $t \in \mathbb{Z}_{+}$. Define the system fundamental matrix $X(t)=A(t-1) \ldots A(0)$ and the monodromy matrix $U=$ $X(\tau)$. Obviously $X(t+\tau)=X(t) U$.

Lemma 2. [27] A periodical system (4) is reducible if $\operatorname{det}\{A(t)\} \neq$ 0 for all $t \in \mathbb{Z}_{+}$.

In this case $N=e^{\tau^{-1} \ln U}$ (i.e. $U=N^{\tau}$ ) and $G(t)=N^{t} X(t)^{-1}$ $\left(G(t)\right.$ is periodical, indeed $G(t+\tau)=N^{t+\tau} X^{-1}(t+\tau)=$ $\left.N^{t} N^{\tau} U^{-1} X^{-1}(t)=N^{t} X^{-1}(t)=G(t)\right)$ [27]. Some solutions also exist in the irreducible case [28].

A canonical representation of the reducible system (4) with possible singular matrices $A(t)$ is studied in [29], where it is shown that any reducible system admits a canonical representation with a matrix $N$ in a Jordan canonical form with only nonnegative eigenvalues (the matrix $N$ is complex in [29], using the transformation proposed in [22] the matrix $N$ can be chosen real).

\section{LTI systems}

In this subsection we briefly review the main result of [21] for an LTI discrete-time system:

$$
x(t+1)=A x(t)+b(t), y(t)=C x(t)+v(t), t \in \mathbb{Z}_{+},
$$

where all variables have the same dimensions and meaning as for the system (1); in this work we assume that $b \in \mathcal{L}_{\infty}, v \in \mathcal{L}_{\infty} ; A$ and $C$ are real matrices with appropriate dimensions.

Assumption 1. Let $x \in \mathcal{L}_{\infty}$ and $\|v\|<V$, the constant $0 \leq V<$ $+\infty$ is known.

Assumption 2. There exists a matrix $L \in \mathbb{R}^{n \times p}$ such that the matrix $A-L C$ is Schur stable.

Assumption 3. There exist two functions $\underline{b}, \bar{b}: \mathbb{Z}_{+} \rightarrow \mathbb{R}^{n}, \underline{b}, \bar{b} \in \mathcal{L}_{\infty}$ such that $\underline{b}(t) \leq b(t) \leq \bar{b}(t)$ for all $\in \mathbb{Z}_{+}$.

Assumption 1 is rather common in the estimation literature. Assumption 2 is standard [3], [1]. Assumption 3 states that the input is not exactly known, but it belongs to the interval $[\underline{b}(t), \bar{b}(t)]$.

Theorem 1. [21] Let assumptions 1-3 be satisfied and there exist a matrix $R \in \mathbb{R}_{+}^{n \times n}$ such that $\lambda(A-L C)=\lambda(R)$ and the pairs $\left(A-L C, \varrho_{1}\right),\left(R, \varrho_{2}\right)$ are observable for some $\varrho_{1} \in \mathbb{R}^{1 \times n}, \varrho_{2} \in$ $\mathbb{R}^{1 \times n}$. Then for all $t \in \mathbb{Z}_{+}$the estimates $\underline{x}(t), \bar{x}(t)$ are bounded and

$$
\underline{x}(t) \leq x(t) \leq \bar{x}(t)
$$

provided that $\underline{x}(0) \leq x(0) \leq \bar{x}(0)$, where $\underline{x}(t)=S^{+} \underline{z}(t)-S^{-} \bar{z}(t)$, $\bar{x}(t)=S^{+} \bar{z}(t)-S^{-} \underline{z}(t)$, and

$$
\begin{aligned}
\underline{z}(t+1)= & R \underline{z}(t)+F y(t)-\bar{F} V \\
& +\left(S^{-1}\right)^{+} \underline{b}(t)-\left(S^{-1}\right)^{-} \bar{b}(t), \\
\bar{z}(t+1)= & R \bar{z}(t)+F y(t)+\bar{F} V \\
& +\left(S^{-1}\right)^{+} \bar{b}(t)-\left(S^{-1}\right)^{-} \underline{b}(t) ; \\
\underline{z}(0)= & \left(S^{-1}\right)^{+} \underline{x}(0)-\left(S^{-1}\right)^{-} \bar{x}(0), \\
\bar{z}(0)= & \left(S^{-1}\right)^{+} \bar{x}(0)-\left(S^{-1}\right)^{-} \underline{x}(0),
\end{aligned}
$$

where $S=O_{R} O_{A-L C}^{-1}\left(O_{A-L C}\right.$ and $O_{R}$ are the observability matrices of the pairs $\left(A-L C, \varrho_{1}\right),\left(R, \varrho_{2}\right)$ respectively), $F=S^{-1} L$ and $\bar{F}=\left(F^{+}+F^{-}\right) E_{p \times 1}$. 
Proof: Consider the system (6), let us add and subtract the signal $L y$, then in the new coordinates $z=S^{-1} x$ we obtain:

$$
\begin{gathered}
z(t+1)=R z(t)+F[y(t)-v(t)]+S^{-1} b(t), \\
y(t)=C S z(t)+v(t) .
\end{gathered}
$$

The dynamics of errors $\underline{e}=z-\underline{z}, \bar{e}=\bar{z}-z$ obey the equations:

$$
\underline{e}(t+1)=R \underline{e}(t)+\underline{d}(t), \quad \bar{e}(t+1)=R \bar{e}(t)+\bar{d}(t),
$$

where $\underline{d}(t)=[\bar{F} V-F v(t)]+\left[S^{-1} b(t)-\left(S^{-1}\right)^{+} \underline{b}(t)+\left(S^{-1}\right)^{-} \bar{b}(t)\right]$, $\bar{d}(t)=[\bar{F} V+F v(t)]+\left[\left(S^{-1}\right)^{+} \bar{b}(t)-\left(S^{-1}\right)^{-} \underline{b}(t)-S^{-1} b(t)\right]$.

By construction the matrix $A-L C$ is Schur stable due to the choice of $L$, then the matrix $R=S^{-1}(A-L C) S$ is Schur stable and nonnegative. Therefore, the variables $\underline{e}(t)$ and $\bar{e}(t)$ are bounded, and taking in mind Assumption 1 we get the boundedness of $\underline{z}(t), \bar{z}(t)$. According to assumptions 1, 3 and the relations (3), we have $\underline{d}, \bar{d} \in$ $\mathcal{L}_{\infty}$ and $\underline{d}(t) \geq 0, \bar{d}(t) \geq 0$ for all $t \in \mathbb{Z}_{+}$. Next, since the matrix $R$ is nonnegative, and $\underline{e}(0) \geq 0, \bar{e}(0) \geq 0$ (by construction $\underline{z}(0) \leq$ $z(0) \leq \bar{z}(0)$ ), then $\underline{e}(t) \geq 0$ and $\bar{e}(t) \geq 0$ for all $t \in \mathbb{Z}_{+}$since $\underline{d}, \bar{d}$ have the same property. This fact implies the required order relation $\underline{z}(t) \leq z(t) \leq \bar{z}(t)$ for all $t \in \mathbb{Z}_{+}$. Finally, using the relations (3) we get the theorem claim that $\underline{x}(t) \leq x(t) \leq \bar{x}(t)$ for all $t \in \mathbb{Z}_{+}$.

If in Assumption 2 we impose observability of the pair $(A, C)$, then formulation of Theorem 1 can be simplified skipping conditions of Lemma 1 (see the discussion after this lemma).

\section{MAIN RESULT}

The main result deals with an extension of Theorem 1 to the LTV case. For this purpose we need to build a transformation of a time-varying matrix to a time-varying nonnegative one. In this work two variants are analyzed: time-invariant and time-varying transformations.

\section{A. Time-invariant similarity transformation}

In this case Assumption 2 is replaced by the following one.

Assumption 4. There exist matrix functions $L: \mathbb{Z}_{+} \rightarrow \mathbb{R}^{n \times p}, P$ : $\mathbb{Z}_{+} \rightarrow \mathbb{R}^{n \times n}, P(\cdot)=P(\cdot)^{\mathrm{T}} \succ 0$ and $\kappa>0$ such that for all $t \in \mathbb{Z}_{+}$:

$$
\begin{gathered}
p_{1} I_{n} \preceq P(t) \preceq p_{2} I_{n}, p_{1}, p_{2}>0 ; \\
(1+\kappa) D(t)^{\mathrm{T}} P(t+1) D(t)-P(t)+Q=0,
\end{gathered}
$$

where $D(t)=A(t)-L(t) C(t)$ and $Q=Q^{\mathrm{T}} \succ 0$.

The condition (9) is satisfied for a matrix $P$ with bounded entries. An approach for such $P$ and $L$ computation can be found in [30], [31]. Next we are going to propose a similarity transformation $S \in \mathbb{R}^{n \times n}$, which maps a stable matrix $D(t)$ to a stable and nonnegative matrix $S^{\mathrm{T}} D(t) S$. For the continuous-time case, a similarity transformation of the matrix $D(t)$ into a Metzler form was developed in [14], the discrete-time version of that result is presented in Lemma 3 below, which needs the following assumption.

Assumption 5. Let $D(t) \in \Xi \subset \mathbb{R}^{n \times n}, \forall t \in \mathbb{Z}_{+}$, where $\Xi=\{D \in$ $\left.\mathbb{R}^{n \times n}: D_{a}-\Delta \leq D \leq D_{a}+\Delta\right\}$ for some $D_{a}^{T}=D_{a} \in \mathbb{R}^{n \times n}$ and $\Delta \in \mathbb{R}_{+}^{n \times n}$. Let $R=\bar{R}^{\mathrm{T}} \geq \mu E_{n \times n}$ be a nonnegative matrix with $\lambda(R)=\lambda\left(D_{a}\right)$ and $\mu>n\|\Delta\|_{\max }$.

Under Assumption 5, if $\|\Delta\|_{\max }$ is sufficiently small, then in Assumption 4 it is possible to look for a solution $P(t)=P$, which can be computed for $D_{a}$ using the robustness theory [32].

Lemma 3. Let Assumption 5 be satisfied, then there exists an orthogonal matrix $S \in \mathbb{R}^{n \times n}$ such that the matrices $S^{T} D S$ are nonnegative for all $D \in \Xi$.
Proof: The matrices $D_{a}$ and $R$ are symmetric due to Assumption 5. Since $\lambda(R)=\lambda\left(D_{a}\right)$ by the same assumption, then there exist two orthogonal matrices $O_{D} \in \mathbb{R}^{n \times n}, O_{R} \in \mathbb{R}^{n \times n}$ such that $O_{D}^{\mathrm{T}} D_{a} O_{D}=O_{R}^{\mathrm{T}} R O_{R}$ (the matrices $O_{D}$ and $O_{R}$ may be composed respectively by eigenvectors of $D_{a}$ and $R$ ). In addition, the matrices $O_{D}$ and $O_{R}$ can be chosen to satisfy $\left\|O_{D}\right\|_{2}=\left\|O_{R}\right\|_{2}=1$. Let $S=O_{D} O_{R}^{\mathrm{T}}$ be another orthogonal matrix with $\|S\|_{2}=1$, then $R=S^{\mathrm{T}} D_{a} S$. For any $D \in \Xi$, we have $S^{\mathrm{T}} D S=S^{\mathrm{T}}\left(D_{a}+\Pi\right) S$ for a matrix $\Pi \in \mathbb{R}^{n \times n}$ satisfying $-\Delta \leq \Pi \leq \Delta$. Then $S^{\mathrm{T}} D S=R+S^{\mathrm{T}} \Pi S$. Using (2) we get $\left\|S^{\mathrm{T}} \Pi S\right\|_{\max } \leq\left\|S^{\mathrm{T}} \Pi S\right\|_{2}=$ $\|\Pi\|_{2} \leq n\|\Pi\|_{\max } \leq n\|\Delta\| \|_{\max }$. Since all elements of the matrix $R$ are bigger than $\mu>n\|\Delta\|_{\max }$, then all elements of the matrix $R+S^{\mathrm{T}} \Pi S$ are nonnegative.

A useful algorithm for a symmetric nonnegative matrix $R$ design is proposed in [26]. The matrix $\mu E_{n \times n}$ has one eigenvalue $\mu n$ and the rests are equal to zero. Since for nonnegative matrices $\mu n=\max \lambda\left(\mu E_{n \times n}\right) \leq \max \lambda(R)$, if $\mu E_{n \times n} \leq R$ [25], and for a Schur stable matrix $R$ we necessarily have $\max \lambda(R) \leq 1$, then we obtain the relation $\mu \leq n^{-1}$, that leads to a restriction $\|\Delta\|_{\max }<n^{-2}$, which has not been met for Metzler matrices in [14]. This restriction means that Lemma 3 can be used if the system uncertainty is not big. Note that this lemma can be applied to systems with an uncertain matrix $D$ since it is based on the average matrix $D_{a}$ and the dispersion $\Delta$.

Under Assumption 5 there is an orthogonal matrix $S \in \mathbb{R}^{n \times n}$ such that $S^{\mathrm{T}} D(t) S \in \mathbb{R}_{+}^{n \times n}$ for all $D(t) \in \Xi$. By introducing a new state variable $z=S^{\mathrm{T}} x$ the system (1) is transformed to

$$
\begin{gathered}
z(t+1)=S^{\mathrm{T}} D(t) S z(t)+F(t)[y(t)-v(t)]+\beta(t), \\
y(t)=C(t) S z(t)+v(t),
\end{gathered}
$$

where $\beta(t)=S^{\mathrm{T}} b(t)$ and $F(t)=S^{\mathrm{T}} L(t)$. Using (3) we have the following relations

$$
\underline{x}=S^{+} \underline{z}-S^{-} \bar{z} \leq x=S z \leq S^{+} \bar{z}-S^{-} \underline{z}=\bar{x},
$$

where $\underline{z} \leq z \leq \bar{z}$ are the interval estimates for the variable $z$. Similarly under assumption 3 we obtain:

$$
\begin{gathered}
\underline{\beta}(t)=S^{+\mathrm{T}} \underline{b}(t)-S^{-\mathrm{T}} \bar{b}(t) \leq \\
\beta(t) \leq S^{+\mathrm{T}} \bar{b}(t)-S^{-\mathrm{T}} \underline{b}(t)=\bar{\beta}(t) .
\end{gathered}
$$

In the new coordinates the observer takes form similar to (8):

$$
\begin{aligned}
& \underline{z}(t+1)=S^{\mathrm{T}} D(t) S \underline{z}(t)+\underline{\beta}(t)-\bar{F}(t) V+F(t) y(t), \\
& \bar{z}(t+1)=S^{\mathrm{T}} D(t) S \bar{z}(t)+\bar{\beta}(t)+\bar{F}(t) V+F(t) y(t),
\end{aligned}
$$

where $\bar{F}(t)=\left[F^{+}(t)+F^{-}(t)\right] E_{p \times 1}$. Now we are in position to prove the following extended variant of Theorem 1 .

Theorem 2. Let assumptions 1, 3-5 be satisfied. Then the variables $\underline{x}(t)$ and $\bar{x}(t)$ in (1), (11), (13) are bounded for all $t \in \mathbb{Z}_{+}$and the relations (7) are satisfied for $\underline{z}(0)=S^{+T} \underline{x}(0)-S^{-T} \bar{x}(0), \bar{z}(0)=$ $S^{+T} \bar{x}(0)-S^{-T} \underline{x}(0)$ provided that $\underline{x}(0) \leq x(0) \leq \bar{x}(0)$.

Proof: Consider the dynamics of the interval estimation errors $\bar{e}=\bar{z}-z, \underline{e}=z-\underline{z}$ :

$\bar{e}(t+1)=S^{\mathrm{T}} D(t) S \bar{e}(t)+\bar{d}(t), \quad \underline{e}(t+1)=S^{\mathrm{T}} D(t) S \underline{e}(t)+\underline{d}(t)$, where $\bar{d}(t)=[\bar{\beta}(t)-\beta(t)]+[\bar{F}(t) V+F(t) v(t)]$ and $\underline{d}(t)=[\beta(t)-$ $\beta(t)]+[\bar{F}(t) V-F(t) v(t)]$. According to assumptions 1, 3 the signals $\overline{\bar{d}}, \underline{d}$ are nonnegative for all $t \in \mathbb{Z}_{+}$and $\underline{d}, \bar{d} \in \mathcal{L}_{\infty}$. By (12) the discrepancies $\bar{\beta}(t)-\beta(t), \beta(t)-\beta(t)$ are positive, and for all $t \geq 0$ we have $\bar{F}(t) V+F(t) v(t)=V\left[\bar{F}^{+}(t)+F^{-}(t)\right] E_{p \times 1}+\left[F^{+}(t)-\right.$ $\left.F^{-}(t)\right] v(t)=F^{+}(t)\left[V E_{p \times 1}+v(t)\right]+F^{-}(t)\left[V E_{p \times 1}-v(t)\right] \geq$ 0 and $\bar{F}(t) V-F(t) v(t)=V\left[F^{+}(t)+F^{-}(t)\right] E_{p \times 1}-\left[F^{+}(t)-\right.$ 
$\left.F^{-}(t)\right] v(t)=F^{+}(t)\left[E_{p \times 1} V-v(t)\right]+F^{-}(t)\left[E_{p \times 1} V+v(t)\right] \geq 0$. By construction $\bar{e}(0) \geq 0, \underline{e}(0) \geq 0$ (from (3)), therefore since $S^{\mathrm{T}} D(t) S \in \mathbb{R}_{+}^{n \times n}$ (from Lemma 3 ) we obtain that $\bar{e}(t) \geq 0, \underline{e}(t) \geq$ 0 for all $t \in \mathbb{Z}_{+}$, which leads to

$$
\underline{z}(t) \leq z(t) \leq \bar{z}(t) \forall t \in \mathbb{Z}_{+}
$$

Finally, the order relation $\underline{x}(t) \leq x(t) \leq \bar{x}(t)$ follows from (3).

To prove boundedness of $\underline{z}(t), \bar{z}(t)(\underline{x}(t)$ and $\bar{x}(t))$ consider the Lyapunov functions $V_{1}(t)=\bar{e}^{\mathrm{T}}(t) S^{\mathrm{T}} P(t) S \bar{e}(t)$ and $V_{2}(t)=$ $\underline{e}^{\mathrm{T}}(t) S^{\mathrm{T}} P(t) S \underline{e}(t)$. Below only $V_{1}$ is analyzed (similarly for $V_{2}$ ):

$$
\begin{gathered}
V_{1}(t+1)-V_{1}(t)=\bar{e}^{\mathrm{T}}(t) S^{\mathrm{T}} D^{\mathrm{T}}(t) P(t+1) D(t) S \bar{e}(t)+ \\
2 \bar{d}^{\mathrm{T}}(t) S^{\mathrm{T}} P(t+1) D(t) S \bar{e}(t)+\bar{d}^{\mathrm{T}}(t) S^{\mathrm{T}} P(t+1) S \bar{d}(t) \\
-\bar{e}^{\mathrm{T}}(t) S^{\mathrm{T}} P(t) S \bar{e}(t) \\
\leq \bar{e}^{\mathrm{T}}(t) S^{\mathrm{T}}\left[(1+\kappa) D^{\mathrm{T}}(t) P(t+1) D(t)-P(t)\right] S \bar{e}(t) \\
+\left(\kappa^{-1}+1\right) p_{2}\|\bar{d}\|^{2} \\
\leq-\bar{e}^{\mathrm{T}}(t) S^{\mathrm{T}} Q S \bar{e}(t)+\left(\kappa^{-1}+1\right) p_{2}\|\bar{d}\|^{2},
\end{gathered}
$$

that gives boundedness of $\underline{z}, \bar{z}\left(p_{2}, \kappa\right.$ come from Assumption 4).

This theorem proposes an interval observer for an LTV system explicitly skipping the requirement on positivity of the closed loop matrix $D$ (according to assumption 4 it is only stable).

In addition, if the system state is nonnegative (that is a usual situation in many biological applications), then it is possible to design the interval observer without any knowledge of the exact values of the matrix function $D$. Under these conditions the interval observers can be also applied for uncertain time-varying systems. This result is formulated below as a corollary of Theorem 2 .

Corollary 1. Let assumptions 1,3 and 5 be satisfied, $\underline{\Pi} \leq$ $S^{\mathrm{T}} D(t) S \leq \bar{\Pi}$ for Schur stable matrices $\underline{\Pi}, \bar{\Pi}$, and $z(t) \in \mathbb{R}_{+}^{n}$ in (10) for all $t \in \mathbb{Z}_{+}$. Consider the interval observer:

$$
\begin{aligned}
& \underline{z}(t+1)=\underline{\Pi} \underline{z}(t)+\underline{\beta}(t)-\bar{F}(t) V+F(t) y(t), \\
& \bar{z}(t+1)=\bar{\Pi} \bar{z}(t)+\bar{\beta}(t)+\bar{F}(t) V+F(t) y(t) .
\end{aligned}
$$

Then the variables $\underline{x}(t)$ and $\bar{x}(t)$ in (1), (11), (14) are bounded for all $t \in \mathbb{Z}_{+}$and (7) is satisfied for $\underline{z}(0)=S^{+T} \underline{x}(0)-S^{-T} \bar{x}(0)$, $\bar{z}(0)=S^{+T} \bar{x}(0)-S^{-T} \underline{x}(0)$ provided that $\underline{x}(0) \leq x(0) \leq \bar{x}(0)$.

Proof: According to conditions of Theorem 2 and Assumption 1 , the system (14) is stable linear time-invariant with bounded inputs, therefore the variables $\underline{z}(t), \bar{z}(t)$ (and hence $\underline{x}(t), \bar{x}(t)$ from $(11)$ ) are bounded. The dynamics of the interval estimation errors $\bar{e}=\bar{z}-z$, $\underline{e}=z-\underline{z}$ can be written as follows:

$$
\bar{e}(t+1)=\bar{\Pi} \bar{e}(t)+\bar{d}(t), \quad \underline{e}(t+1)=\underline{\Pi e}(t)+\underline{d}(t),
$$

where $\bar{d}(t)=[\bar{\beta}(t)-\beta(t)]+[\bar{F}(t) V+F(t) v(t)]+[\bar{\Pi}-$ $\left.S^{\mathrm{T}} D(t) S\right] z(t), \underline{d}(t)=[\beta(t)-\underline{\beta}(t)]+[\bar{F}(t) V-F(t) v(t)]+$ $\left[S^{\mathrm{T}} D(t) S-\underline{\Pi}\right] z(t)$ are positive inputs. Since the matrices $\underline{\Pi}, \bar{\Pi}$ are nonnegative by Assumption 5 and $\bar{e}(0) \geq 0, \underline{e}(0) \geq 0$, then $\bar{e}(t) \geq 0, \underline{e}(t) \geq 0$ for all $t \in \mathbb{Z}_{+}$.

\section{B. Time-varying transformation}

The objective of this subsection is to build a time-varying transformation of coordinates $z(t)=G^{-1}(t) x(t)$ with $\operatorname{det}\{G(t)\} \neq 0$ for all $t \in \mathbb{Z}_{+}$and $\sup _{t \in \mathbb{Z}_{+}}\|G(t)\|_{2}+\left\|G^{-1}(t)\right\|_{2}<+\infty$, which maps the system (1) to the following one:

$$
\begin{gathered}
z(t+1)=R(t) z(t)+G^{-1}(t+1)[b(t)+L(t)\{y(t)-v(t)\}], \\
y(t)=C(t) G(t) z(t)+v(t), t \in \mathbb{Z}_{+},
\end{gathered}
$$

where $R(t)=G^{-1}(t+1)[A(t)-L(t) C(t)] G(t)$ should be a stable and nonnegative matrix, and $L: \mathbb{Z}_{+} \rightarrow \mathbb{R}^{n \times p}$ is an observer gain matrix to be calculated. Therefore, in the common case the transformation matrix $G$ is a solution of the following discrete-time dynamical equation:

$$
G(t+1) R(t)=D(t) G(t), t \in \mathbb{Z}_{+}
$$

with an initial condition $G(0) \in \mathbb{R}^{n \times n}$ and $D(t)=A(t)-L(t) C(t)$. For an invertible matrices $R$, using the Kronecker product $\otimes$, this equation can be rewritten as follows:

$$
\begin{aligned}
\operatorname{vec}\{G(t+1)\} & =\operatorname{vec}\left\{D(t) G(t) R(t)^{-1}\right\} \\
& =\Gamma(t) \operatorname{vec}\{G(t)\}, \\
\Gamma(t) & =\left[R(t)^{-1}\right]^{\mathrm{T}} \otimes D(t),
\end{aligned}
$$

where $\operatorname{vec}\{S\}=\left[S_{1,1}, \ldots S_{m, 1}, S_{2,1} \ldots S_{n, n}\right]^{\mathrm{T}}$ is the vectorization transformation of a matrix $S \in \mathbb{R}^{n \times n}$, and $\lambda[\Gamma(t)]=\lambda\left[R(t)^{-1}\right] \otimes$ $\lambda[D(t)]$. Thus it is necessary to select the matrices $L(t)$ and $R(t)$ in a way that the system (15) has a bounded solution $G(t)$ with a bounded inverse $G^{-1}(t)$, which is a hardly solving problem. An admissible solution can be an oscillating bounded matrix $G(t)$, which is the case of periodical systems, for example.

Further this subsection deals with periodical systems (1), i.e. we will assume that there is a $\tau>1$ such that $A(t+\tau)=A(t)$, $C(t+\tau)=C(t)$ for all $t \in \mathbb{Z}_{+}$.

Assumption 6. There exists a matrix function $L: \mathbb{Z}_{+} \rightarrow \mathbb{R}^{n \times p}$ such that for all $t \in \mathbb{Z}_{+}$:

i) $L(t+\tau)=L(t)$;

ii) $\operatorname{det}\{D(t)\} \neq 0, D(t)=A(t)-L(t) C(t)$;

iii) $N=e^{\tau^{-1} \ln U}$ is Schur stable and nonnegative, where $U=$ $X(\tau), X(t)=D(t) \ldots D(0)$.

This is a standard assumption [28]. By adding and subtracting $L(t) y(t)$ in (1) we obtain:

$$
x(t+1)=D(t) x(t)+L(t)[y(t)-v(t)]+b(t) .
$$

Under Assumption 6 this system is reducible via the coordinate transformation $z(t)=G^{-1}(t) x(t), G(t)=N^{t} X(t)^{-1}$ to the following cooperative system:

$$
z(t+1)=N z(t)+F(t)[y(t)-v(t)]+\beta(t),
$$

where $F(t)=G^{-1}(t+1) L(t)$ is a periodical matrix and $\beta(t)=$ $G^{-1}(t+1) b(t)$.

Note that the part (iii) of Assumption 6 can be relaxed assuming that $N$ is only Schur stable. Thereby if there exists a matrix $R \in$ $\mathbb{R}_{+}^{n \times n}$ such that $\lambda(N)=\lambda(R)$ and the pairs $\left(N, \varrho_{1}\right),\left(R, \varrho_{2}\right)$ are observable for some $\varrho_{1} \in \mathbb{R}^{1 \times n}, \varrho_{2} \in \mathbb{R}^{1 \times n}$ then according to Lemma 1 there is an invertible matrix $S \in \mathbb{R}^{n \times n}$ such that the conditions of Assumption 6 are satisfied for $\widetilde{N}=R$ and $\widetilde{G}(t)=$ $S G(t)$. Another way to relax the part (iii) of Assumption 6 is to modify the time-varying transformation $G(t)$ using the time-varying transformation proposed in [22], that maps a time-invariant matrix to a nonnegative one.

Finally, the interval observer for the system (16) has the form:

$$
\begin{aligned}
& \underline{z}(t+1)=N \underline{z}(t)+F(t) y(t)-\bar{F}(t) V+\underline{\beta}(t), \\
& \bar{z}(t+1)=N \bar{z}(t)+F(t) y(t)+\bar{F}(t) V+\bar{\beta}(t),
\end{aligned}
$$

where $\bar{F}(t)=\left[F^{+}(t)+F^{-}(t)\right] E_{p \times 1}$ and from (3) we obtain

$$
\begin{gathered}
\underline{\beta}(t)=\left[G^{-1}(t+1)\right]^{+} \underline{b}(t)-\left[G^{-1}(t+1)\right]^{-} \bar{b}(t) \leq \beta(t) \\
\leq\left[G^{-1}(t+1)\right]^{+} \bar{b}(t)-\left[G^{-1}(t+1)\right]^{-} \underline{b}(t)=\bar{\beta}(t) .
\end{gathered}
$$

Theorem 3. Let assumptions 1,3 and 6 be satisfied. Then for all $t \in \mathbb{Z}_{+}$the estimates

$\underline{x}(t)=G^{+}(t) \underline{z}(t)-G^{-}(t) \bar{z}(t), \quad \bar{x}(t)=G^{+}(t) \bar{z}(t)-G^{-}(t) \underline{z}(t)$ 
are bounded for (1), (17) and the relations (7) are satisfied for all $t \in \mathbb{Z}_{+}$with $\underline{z}(0)=\left[G^{-1}(t)\right]^{+} \underline{x}(0)-\left[G^{-1}(t)\right]^{-} \bar{x}(0), \bar{z}(0)=$ $\left[G^{-1}(t)\right]^{+} \bar{x}(0)-\left[G^{-1}(t)\right]^{-} \underline{x}(0)$ provided that $\underline{x}(0) \leq x(0) \leq \bar{x}(0)$. proof.

Proof: The proof essentially repeats the steps of Theorem 2

For the case of periodical systems this theorem is less restrictive than Theorem 2, where implicitly $\|\Delta\|_{\max }<n^{-2}$ is assumed (the deviation of $D(t)$ from its average has to be sufficiently small).

Remark 1. It is worth to stress that $b$ or $\beta$ could be a function of the state $x$ provided that there exist known bounded signals $\bar{b}, \underline{b}$ satisfying Assumption 3. Similarly for the matrix functions $A$ and $C$, they can be nonlinear functions of the system input/output variables available for measurements. Therefore, the presented interval observers (8), (13), (17) can be applied to nonlinear systems in the output canonical form, for instance. A mild reformulation of theorems 1-3 for this case is skipped for brevity of exposition. Application of these theorems to nonlinear systems is illustrated on examples in Section 4.

\section{EXAMPLES}

To illustrate the proposed results consider two examples.

\section{A. Repressilator system}

Following [33], [34] consider a model of an oscillatory network of transcriptional regulators with three genes:

$$
\begin{aligned}
\dot{m}_{i} & =-m_{i}+\frac{\alpha(t)}{1+p_{i-1}^{n}}+\alpha_{i}^{0}(t), \\
\dot{p}_{i} & =\nu(t) m_{i}-\mu(t) p_{i}, i=1,2,3,
\end{aligned}
$$

where the convention $p_{0}=p_{3}$ is used, $x=$ $\left[m_{1}, p_{1}, m_{2}, p_{2}, m_{3}, p_{3}\right] \in \mathbb{R}_{+}^{6}$ is the state, $m_{i}$ represents the mRNA concentration of a gene, $p_{i}$ is the corresponding protein concentration. In this work (contrarily to [33], [34]) it is assumed that all parameters are functions of time:

$$
\begin{gathered}
\underline{\alpha} \leq \alpha(t) \leq \bar{\alpha}, \underline{\nu} \leq \nu(t) \leq \bar{\nu}, \underline{\mu} \leq \mu(t) \leq \bar{\mu} \\
\underline{\alpha}^{0} \leq \alpha_{i}^{0}(t) \leq \bar{\alpha}^{0}, i=1,2,3 ; n=1,
\end{gathered}
$$

and only the bounds $\underline{\alpha}=0.5, \bar{\alpha}=1.5, \underline{\nu}=1.9, \bar{\nu}=2.1, \mu=1.9$, $\bar{\mu}=2.1, \underline{\alpha}^{0}=25, \bar{\alpha}^{0}=35$ are available for estimation. Such an assumption allows us to simulate a larger class of uncertainties presented in the repressilator model. For simulation we will use

$$
\begin{gathered}
\alpha(t)=1+0.5 \cos (0.2 t), \nu(t)=2+0.1 \sin (0.1 t), \\
\mu(t)=2+0.1 \sin (0.2 t), \\
\alpha_{1}^{0}(t)=30+3 \cos (0.4 t)+2 \sin (0.3 t), \\
\alpha_{2}^{0}(t)=30-3 \cos (0.3 t)+2 \sin (0.4 t), \\
\alpha_{3}^{0}(t)=30+3 \cos (0.3 t)-2 \sin (0.4 t) .
\end{gathered}
$$

The values of $m_{1}, m_{2}, m_{3}$ are available for measurements at the discrete instants of time with the period $T=5$ [min].

In order to apply the proposed theoretical results we discretize the model with the period of measurements $T$ using the Euler method for all $i=1,2,3\left(t_{k}=T k, k \in \mathbb{Z}_{+}\right)$:

$$
\begin{gathered}
{\left[\begin{array}{c}
m_{i}(k+1) \\
p_{i}(k+1)
\end{array}\right]=A\left(t_{k}\right)\left[\begin{array}{c}
m_{i}(k) \\
p_{i}(k)
\end{array}\right]+\beta_{i}\left(t_{k}, p_{i-1}(k)\right),} \\
A(t)=\left[\begin{array}{cc}
1-T & 0 \\
T \nu(t) & 1-T \mu(t)
\end{array}\right], \beta_{i}(t, p)=T\left[\begin{array}{c}
\frac{\alpha(t)}{1+p^{n}}+\alpha_{i}^{0}(t) \\
0
\end{array}\right] .
\end{gathered}
$$

Assumption 1 is satisfied with $V=0$ (it is assumed that there is no measurement noise). The only nonlinear term $\beta_{i}$ is always bounded by construction for positive concentrations $p_{i-1}$ (confirming

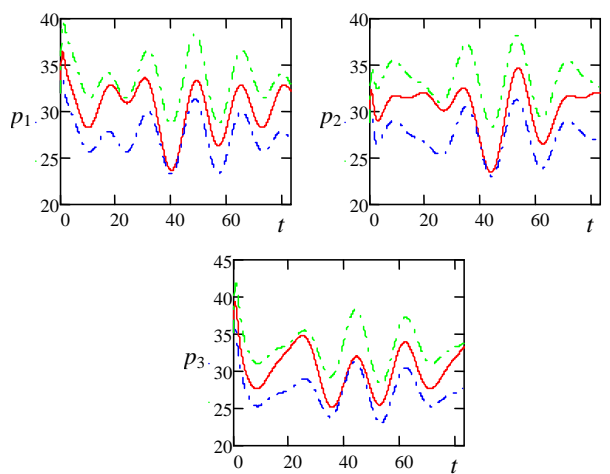

Figure 1. The protein concentration interval estimation

Remark 1). In addition, the time-varying matrix $A(t)$ is nonnegative (Assumption 5 is satisfied). For each $i=1,2,3$ we have the following overlaying and underlying matrices:

$$
\begin{gathered}
\underline{A} \leq A(t) \leq \bar{A} \\
\underline{A}=\left[\begin{array}{cc}
1-T & 0 \\
T \underline{\nu} & 1-T \bar{\mu}
\end{array}\right], \bar{A}=\left[\begin{array}{cc}
1-T & 0 \\
T \bar{\nu} & 1-T \underline{\mu}
\end{array}\right] .
\end{gathered}
$$

For $L=\left[\begin{array}{ll}0.9 & 0.15\end{array}\right]^{\mathrm{T}}$ and $C=\left[\begin{array}{ll}1 & 0\end{array}\right]$ the matrices $\underline{\Pi}=\underline{A}-L C$ and $\bar{\Pi}=\bar{A}-L C$ are Schur stable and nonnegative, the state is positive and Assumption 3 is satisfied for

$$
\begin{aligned}
& \underline{\beta}(k)=T\left[\begin{array}{c}
\frac{\alpha}{1+\max \left\{0, \bar{p}^{n}(k)\right\}}+\underline{\alpha}^{0} \\
0
\end{array}\right], \\
& \bar{\beta}(k)=T\left[\begin{array}{c}
\frac{\alpha}{1+\max \left\{0, \underline{p}^{n}(k)\right\}}+\bar{\alpha}^{0} \\
0
\end{array}\right] .
\end{aligned}
$$

All conditions of Corollary 1 are valid. The results of simulation for the obtained interval observer (14) are presented in Fig. 1 for the unmeasured concentrations $p_{i}$.

\section{B. Time-varying nonlinear system}

Consider a third order discrete-time system:

$$
\begin{aligned}
& x(t+1)=A(t) x(t)+\phi(y(t))+d(t)+\delta d(t), y(t)=x_{1}(t), \\
& A(t)=\left[\begin{array}{ccc}
1.1 & \delta \cos (2 \omega t)-0.1 & r-\delta \sin (0.5 \omega t) \\
0.9+\delta \cos (\omega t) & a-\delta \sin (0.5 \omega t) & \delta \sin (\omega t)-a \\
0.85 & \delta \cos (\omega t)-a & 0.25
\end{array}\right] \text {, } \\
& \phi(y)=\left[-0.1|y|^{2} \operatorname{sign}(y) 05 \sin (y)\right]^{\mathrm{T}}, \delta=0.05, \omega=\pi / 10, \\
& d(t)=[\sin (\omega t)-\cos (0.5 \omega t) \sin (2 \omega t)]^{\mathrm{T}},
\end{aligned}
$$

where $\|\delta d\| \leq \overline{\delta d}=0.5$ is an uncertain input (for simulation $\delta d(t)=$ $\left.0.5[-\sin (2 \omega t) \cos (\omega t)-\sin (4 \omega t)]^{\mathrm{T}}\right), a=0.2, r=0.35$. Numerical simulations show that for initial conditions $|x(0)| \leq 1$ the system has bounded trajectories despite of instability of $A(t)$ (Assumption 1 is satisfied). Define $b(t)=\phi(y(t))+d(t)+\delta d(t)$, then the system can be rewritten in the form (1) and Assumption 3 holds with

$\underline{b}(t)=\phi(y(t))+d(t)-\overline{\delta d} E_{3 \times 1}, \bar{b}(t)=\phi(y(t))+d(t)+\overline{\delta d} E_{3 \times 1}$.

The matrix $A(t)$ is periodical, but the results of Theorem 3 cannot be applied here since the monodromy matrix $U$ is very close to a singular (the inversion of $U$ needed in Theorem 3 leads to a high numerical error of computations). From another side, $A(t)$ belongs to the interval $\left[A_{a}-\Delta, A_{a}+\Delta\right]$, where

$$
A_{a}=\left[\begin{array}{ccc}
1.1 & -0.1 & r \\
0.9 & a & -a \\
0.85 & -a & 0.25
\end{array}\right], \Delta=\left[\begin{array}{lll}
0 & \delta & \delta \\
\delta & \delta & \delta \\
0 & \delta & 0
\end{array}\right]
$$



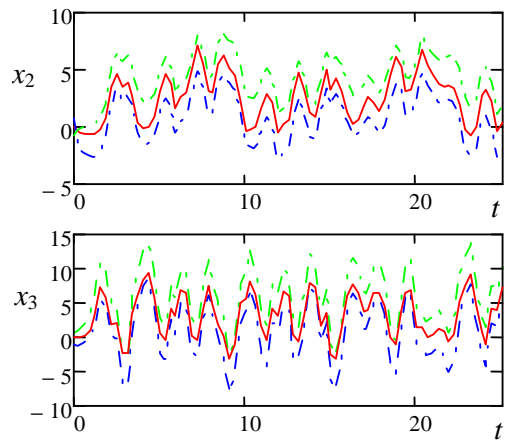

Figure 2. The results of interval estimation for a time-varying nonlinear system

For $L=\left[\begin{array}{lll}1 & 1 & 0.5\end{array}\right]^{\mathrm{T}}$ the matrix $D_{a}=A_{a}-L C$ becomes Schur stable (it is straightforward to verify that Assumption 4 is true for $P=I_{3}$ ), but there is no possibility to make it nonnegative for any $L$. Thus an orthonormal transformation

$$
S=\left[\begin{array}{ccc}
-0.058 & 0.997 & -0.052 \\
0.134 & -0.044 & -0.99 \\
0.989 & 0.064 & 0.131
\end{array}\right]
$$

is calculated such that

$$
R=S^{\mathrm{T}} D_{a} S=\left[\begin{array}{lll}
0.157 & 0.346 & 0.173 \\
0.346 & 0.156 & 0.163 \\
0.173 & 0.163 & 0.237
\end{array}\right]
$$

is a nonnegative matrix with $\mu=0.156\left(\|\Delta\|_{\max }=\delta\right)$ and Assumption 5 is satisfied. Therefore, all conditions of Theorem 2 are verified, the results of interval estimation using (13) for the unmeasured coordinates $x_{2}$ and $x_{3}$ are shown in Fig. 2 .

\section{CONCLUSION}

The paper presents a new solution for designing an interval observer for time-varying discrete-time systems. The approach can be also applied to nonlinear systems in the output canonical form (like in the repressilator example). Static and dynamic transformations of coordinates are proposed mapping a stable LTV system to another LTV system that is stable and cooperative. Simulation results are provided to show the efficiency of the proposed method.

With respect to already existing design methods for continuoustime systems, the paper has demonstrated that the discrete-time interval observers admit a relaxation of some applicability conditions. The relaxation of symmetry of the matrix $D_{a}$ introduced in the conditions of the Lemma 3 and the stability conditions used in Assumption 4 are future directions of research. This would provide possibility for an extension of the proposed techniques to more complex systems.

\section{REFERENCES}

[1] T. Meurer, K. Graichen, and E.-D. Gilles, Eds., Control and Observer Design for Nonlinear Finite and Infinite Dimensional Systems. Springer, 2005, vol. Lecture Notes in Control and Information Sciences, no. 322.

[2] H. Nijmeijer and T. I. Fossen, New Directions in Nonlinear Observer Design. London, U.K.: Springer-Verlag, 1999.

[3] G. Besançon, Ed., Nonlinear observers and applications, ser. Lecture Notes in Control and Inforamtion Science. Berlin: Springer Verlag, 2007, vol. 363.

[4] J. Tsinias, "Time-varying observers for a class of nonlinear systems," System and Control Letters, vol. 57, pp. 1037-1047, 2008.

[5] D. Efimov, A. Zolghadri, and P. Simon, "Improving fault detection abilities of extended kalman filters by covariance matrices adjustment," in Proc. IEEE SysTol 2010, Nice, 2010.
[6] K. Reif and R. Unbehauen, "The extended kalman filter as an exponential observer for nonlinear systems," IEEE Transactions on Signal Processing, vol. 47, no. 8, pp. 2324-2328, 1999.

[7] O. Bernard and J. L. Gouzé, "Closed loop observers bundle for uncertain biotechnological models." J. Process Control, vol. 14, pp. 765-774, 2004.

[8] M. Moisan, O. Bernard, and J. L. Gouzé, "Near optimal interval observers bundle for uncertain bio-reactors," Automatica, vol. 45, no. 1, pp. 291-295, 2009.

[9] F. Mazenc and O. Bernard, "Asymptotically stable interval observers for planar systems with complex poles," IEEE Trans. Autom. Control, vol. 55, no. 2, pp. 523-527, 2010.

[10] T. Raïssi, D. Efimov, and A. Zolghadri, "Interval state estimation for a class of nonlinear systems," IEEE Trans. Automatic Control, vol. 57, no. 1 , pp. 260-265, 2012.

[11] D. Efimov, L. Fridman, T. Raïssi, A. Zolghadri, and R. Seydou, "Interval estimation for lpv systems applying high order sliding mode techniques," Automatica, vol. 48, pp. 2365-2371, 2012.

[12] D. Efimov, T. Raïssi, and A. Zolghadri, "Control of nonlinear and lpv systems: interval observer-based framework," IEEE Trans. Automatic Control, vol. 58, no. 3, pp. 773-782, 2013.

[13] F. Mazenc and O. Bernard, "Interval observers for linear time-invarian systems with disturbances," Automatica, vol. 47, pp. 140-147, 2011.

[14] D. Efimov, T. Raïssi, S. Chebotarev, and A. Zolghadri, "Interval state observer for nonlinear time varying systems," Automatica, vol. 49, no. 1 pp. 200-205, 2013

[15] L. Jaulin, M. Kieffer, O. Didrit, and E. Walter, Applied Interval Analysis with Examples in Parameter and State Estimation, Robust Control and Robotics. Springer-Verlag, 2001.

[16] L. Chisci, A. Garulli, and G. Zappa, "Recursive state bounding by parallelotopes," Automatica, vol. 32, no. 7, pp. 1049 - 1055, 1996.

[17] E. Walter, J. Norton, H. Piet-Lahanier, and M. Milanese, Bounding Approaches to System Identification. Perseus Publishing, 1996.

[18] D. G. Maksarov and J. P. Norton, "Computationally efficient algorithms for state estimation with ellipsoidal approximations," Int. J. Adaptive Control and Signal Processing, vol. 16, no. 6, pp. 411-434, 2002.

[19] B. T. Polyak, S. A. Nazin, C. Durieu, and E. Walter, "Ellipsoidal parameter or state estimation under model uncertainty," Automatica vol. 40, no. 7, pp. 1171-1179, 2004.

[20] T. Alamo, J. M. Bravo, and E. F. Camacho, "Guaranteed state estimation by zonotopes," Automatica, vol. 41, no. 6, pp. 1035-1043, 2005.

[21] D. Efimov, W. Perruquetti, T. Raïssi, and A. Zolghadri, "On interval observer design for discrete systems," in Proc. ECC 2013, 2013.

[22] F. Mazenc, T. N. Dinh, and S. I. Niculescu, "Interval observers for discrete-time systems," in Proc. IEEE CDC 2012, Maui, Hawaii, 2012, pp. 6755-6760.

[23] M. W. Hirsch and H. L. Smith, "Monotone maps: a review," J. Difference Equ. Appl., vol. 11, no. 4-5, pp. 379-398, 2005.

[24] R. H. Bartels and G. W. Stewart, "Solution of the matrix equation ax + $\mathrm{xb}=\mathrm{c}$, " Comm. ACM, vol. 15, pp. 820-826, 1972.

[25] A. Berman and R. J. Plemmons, Nonnegative matrices in the mathematical sciences, ser. Classics in applied mathmatics. Philadelphia: SIAM, 1994.

[26] O. Rojo and H. Rojo, "Constructing symmetric nonnegative matrices via the fast fourier transform," Computers and Mathematics with Applications, vol. 45, pp. 1655-1672, 2003.

[27] A. Khalanai and D. Veksler, Qualitative Theory of Sampled-Data Systems. Moscow: Mir, 1971.

[28] S. Bittanti and P. Colaneri, Periodic Systems: Filtering and Control, ser. Communications and Control Engineering. Springer, 2008.

[29] I. V. Gaishun, "The canonical form of reducible discrete systems with degenerate coefficient matrices," Differential Equations, vol. 36, no. 7, pp. 1043-1049, 2000.

[30] G. Millerioux, L. Rosier, G. Bloch, and J. Daafouz, "Bounded state reconstruction error for lpv systems with estimated parameters," IEEE Transactions on Automatic Control, vol. 49, no. 8, pp. 1385-1389, 2004.

[31] W. Heemels, J. Daafouz, and G. Millerioux, "Observer-based control of discrete-time lpv systems with uncertain parameters," IEEE Transactions on Automatic Control, vol. 55, no. 9, pp. 2130-2135, 2010.

[32] I. Konstantopoulos and P. Antsaklis, "New bounds for robust stability of continuous and discrete-time systems under parametric uncertainty," Kybernetika, vol. 31, no. 6, pp. 623-636, 1995.

[33] M. B. Elowitz and S. Leibler, "A synthetic oscillatory network of transcriptional regulators," Nature, vol. 403, pp. 335-338, 2000.

[34] G. Lillacci and M. Khammash, "Parameter estimation and model selection in computational biology," PLoS Comput Biol, vol. 6, no. 3, p. e1000696, 2010. 